\title{
Annotation and sequence diversity of transposable elements in common bean (Phaseolus vulgaris)
}

\author{
Dongying Gao ${ }^{1}$, Brian Abernathy ${ }^{1}$, Daniel Rohksar ${ }^{2}$, Jeremy Schmutz ${ }^{2,3}$ and Scott A. Jackson ${ }^{\text {* }}$ \\ ' Center for Applied Genetic Technologies, University of Georgia, Athens, GA, USA \\ 2 US Department of Energy Joint Genome Institute, Walnut Creek, CA, USA \\ ${ }^{3}$ HudsonAlpha Institute of Biotechnology, Huntsville, AL, USA
}

\author{
Edited by: \\ Rajeev K. Varshney, International \\ Crops Research Institute for the \\ Semi-Arid Tropics, India \\ Reviewed by: \\ Eduard Akhunov, Kansas State \\ University, USA \\ Kyujung Van, Seoul National \\ University, South Korea \\ ${ }^{*}$ Correspondence: \\ Scott A. Jackson, Center for Applied \\ Genetic Technologies, University of \\ Georgia, 111 Riverbend Road, Athens, \\ GA 30602, USA \\ e-mail: sjackson@uga.edu
}

\begin{abstract}
Common bean (Phaseolus vulgaris) is an important legume crop grown and consumed worldwide. With the availability of the common bean genome sequence, the next challenge is to annotate the genome and characterize functional DNA elements. Transposable elements (TEs) are the most abundant component of plant genomes and can dramatically affect genome evolution and genetic variation. Thus, it is pivotal to identify TEs in the common bean genome. In this study, we performed a genome-wide transposon annotation in common bean using a combination of homology and sequence structure-based methods. We developed a 2.12-Mb transposon database which includes 791 representative transposon sequences and is available upon request or from www.phytozome.org. Of note, nearly all transposons in the database are previously unrecognized TEs. More than 5,000 transposon-related expressed sequence tags (ESTs) were detected which indicates that some transposons may be transcriptionally active. Two Ty1-copia retrotransposon families were found to encode the envelope-like protein which has rarely been identified in plant genomes. Also, we identified an extra open reading frame (ORF) termed ORF2 from 15 Ty3-gypsy families that was located between the ORF encoding the retrotransposase and the $3^{\prime}$ LTR. The ORF2 was in opposite transcriptional orientation to retrotransposase. Sequence homology searches and phylogenetic analysis suggested that the ORF2 may have an ancient origin, but its function is not clear. These transposon data provide a useful resource for understanding the genome organization and evolution and may be used to identify active TEs for developing transposon-tagging system in common bean and other related genomes.
\end{abstract}

\section{Keywords: transposon, common bean, transposon database, evolution, ORF2}

\section{INTRODUCTION}

Large portions of all sequenced plant genomes consist of highly repetitive sequences, such as transposable elements (TEs) and tandem repeats, which play crucial roles in plant genome organization. In contrast to other repetitive sequences, TEs are mobile genetic elements that can move within genome or via horizontal transfer between genomes (Roulin et al., 2008). In some cases, TE insertions can lead to deleterious or lethal effects to the organism in which they reside. TEs can impact genome structure and evolution. For example, centromeric retrotransposons (CRs) may be involved in the formation of functional centromeres (Jin et al., 2004). TEs serve as important components of heterochromatin maintaining chromosome stability and heterochromatic silencing (Grewal and Jia, 2007). TEs provide raw material for evolutionary novelties, such as new gene functions, expression patterns, and networks (Cordaux and Batzer, 2009). In addition, TEs have been used as mutagens to isolate genes and characterize biological functions.

Transposable elements are divided into two classes based on their transposition mechanisms: class 1 retrotransposons and class 2 DNA transposons. Class 1 incudes long terminal repeats (LTR) retrotransposons and non-LTR retrotransposons including long interspersed elements (LINEs) and short interspersed elements (SINEs). Class 2 contains the elements with terminal inverted repeats (TIRs) and internal sequences that encode enzymes necessary for movements (Wicker et al., 2007). Except for Helitron transposons (Class 2), transposition of all TEs generate target site duplications (TSDs) that range in size from 2-bp to more than 20-bp, according to TE superfamilies.

Transposable elements contribute significant fractions of many eukaryotic genomes and can dramatically impact genome structure, thus annotating TEs is a necessary activity (Juretic et al., 2004). Software tools have been developed to aid in the discovery of TEs based on different annotation strategies. RepeatMasker ${ }^{1}$ is widely used to annotate TEs, however, it depends on a userdefined TE library, the completeness of which affects annotation accuracy and efficiency. RECON was developed for de novo identification of TEs with multiple copies in genome (Bao and Eddy, 2002), thus low-copy TEs may be missed. TEs have structural features, including LTRs, TIRs, and TSDs, that can be used to distinguish them from genes and other genomic features. Several tools have been developed using structural features including

${ }^{1}$ http://www.repeatmasker.org 
LTR_STRUC (McCarthy and McDonald, 2003), LTR_FINDER (Xu and Wang, 2007), MITEs-Hunter (Han and Wessler, 2010) and others (Bergman and Quesneville, 2007). These programs predict intact TEs and truncated TEs may be missed. Another method to find TEs is based on homology. Autonomous TEs encode transposases which are relatively conserved within a transposon superfamily (Wicker et al., 2007), thus the conserved domains of transposases from different superfamilies can be used to search the genome and identify related TEs.

Common bean (Phaseolus vulgaris L., $2 n=2 x=22$ ) is an important food crop grown and consumed throughout the world and can be a major source of income for small farmers in some parts of the world (Blair et al., 2011). Common bean provides protein, fiber, micronutrients, and other valuable components that are absent or in low amounts in other crops and has been referred to as the "healthy people's meat." Common bean belongs to the legume family which is most notable for its ability to fix atmospheric nitrogen via symbioses with soil-borne rhizobia thereby reducing fertilizer costs for farmers. As a close relative of soybean (Glycine max, $2 n=2 x=40$ ), common bean has been used to study domestication, effects of polyploidy and genome evolution of legumes (Lin et al., 2010; McClean et al., 2010; Bitocchi et al., 2012).

Genome sequences for soybean and pigeon pea (Cajanus cajan) have been reported (Schmutz et al., 2010; Varshney et al., 2011). As an important crop and a relative of these two sequenced genomes, the 521.1-Mbp common bean genome has been sequenced (Schmutz et al., 2014). In this study, we conducted genome-wide transposon annotation and developed a common bean transposon database. More than 5,000 transposon-related ESTs were found and suggesting that some TEs in common bean are transcriptionally active. We analyzed the sequence structures of LTR retrotransposons and identified 17 families that contain either the envelope-like protein or an additional open reading frame (ORF2) encoding an unknown protein. Our transposon database provides a valuable resource for the common bean genome annotation and comparative genomics within legumes, and may be used to discover active TEs for transposon-tagging in common bean.

\section{RESULTS}

\section{CONSTRUCTION OF A TRANSPOSON DATABSASE FOR COMMON BEAN}

Several approaches were employed to discover transposon sequences in the common bean genome. First, sequences for 15 BACs $(2.2 \mathrm{Mb})$ were obtained from GenBank and screened with LTR_FINDER to develop a control set of transposons. In addition, all against all BLASTN searches were performed for the 15 BAC sequences. A total of 12 LTR retrotransposon families were identified from these BAC sequences. To identify additional LTR retrotransposon in common bean, LTR_FINDER (Xu and Wang, 2007) was used to screen the draft genome sequence of common bean (V1.0 available from www.phytozome.net) that yielded 10,349 potential LTR retroelements. The boundaries, TSDs, structures of all these sequences were manually inspected which resulted in 2,288 sequences being designed as LTR retrotransposons and another 8,061 sequences were discarded as they were either tandem repeats, incomplete transposons or other sequences. The 2,288 elements were classified into 165 distinct families according published criteria (Wicker et al., 2007), this includes the 12 families identified in the 15 BACs and the two previously described LTR retroelements, Tpv2-6 (Garber et al., 1999) and pva1-118d24-re5 (Wawrzynski et al., 2008). To classify these LTR retroelement families, representative elements for each of 165 families were used to conduct BLASTX or/and BLASTP searches. Sixty-five and 78 families were classified into Ty1-copia and Ty3-gypsy group, respectively. We were not able to group the other 22 families as these elements either encoded no retrotransposase or the encoded proteins were very short. Numerous LTR retroelements were found with exactly identical LTRs which indicates recent insertions of these retroelements and that some of them may still be active in common bean.

Long interspersed elementss are ubiquitous components of many characterized organisms. For instance, they are present in more than 500,000 copies in human comprising $\sim 17 \%$ of the genome (Cordaux and Batzer, 2009). LINEs are also abundant in plants and play important role in plant genome evolution (Feschotte et al., 2002). Typical LINEs contain a Poly (A) tail and a reverse transcriptase (RT) that shares little similarity to those of LTR retrotransposons. Additionally, complete LINEs usually flanked by TSDs that vary in length from 5-bp to more than 20-bp (Kojima, 2010). These features can be used to discover the LINEs in common bean. We used the conserved domain of the RT of LINEs (Feschotte etal., 2002) to conduct TBLASTN against the whole genome sequence and the BAC sequences. All hits $\left(E\right.$ value $<10^{-20}$ ) and flanking sequences $(10$-kb for each side) were extracted and used to search the genome and GenBank to identify the poly (A) motif and TSDs. We were able to identify several complete LINEs. For instance, a 12,917-bp LINE named pvLINE1 encodes an endonuclease and retrovirus RT and contains a 35-bp poly (A/T) tail. pvLINE1 was flanked by 29-bp TSD. However, not all LINEs can be defined as complete elements as they may have accumulated mutations and/or internal rearrangements or due to frequent premature arrest of reverse transcription which can result in truncated LINEs at the $5^{\prime}$ end (Brouha et al., 2003; Cordaux and Batzer, 2009).

A total of 12 superfamilies of DNA transposons have been identified across kingdoms of which six superfamilies are found in plants. These include Mutator-like element (MULE), hAT, CACTA, PIF/Harbinger, Helitron, and Tc1/Mariner; elements such as $\mathrm{P}$ elements and PiggyBac are present in animals only or other organisms (Feschotte and Pritham, 2007; Wicker et al., 2007). To detect DNA transposons in common bean, the conserved domains of the six superfamilies were used as queries to conduct TBLASTN searches. Hits were found for MULE, hAT, CACTA, PIF/Harbinger, and Helitron transposons; however, no hit was found for the Tc1/Mariner superfamilies. We then used MITEsHunter (Han and Wessler, 2010) to find DNA transposons without transposases, the output sequences were manually inspected and their classifications were determined according to the terminal repeats and TSDs. For example, CACTA elements were defined by the "CACTA. ..ATGTG" terminal motifs and 2-3 bp TSD. We identified 348 CACTA sequences, 45 MULEs, 23 hATs, and 39 Helitrons. An additional 46 repetitive sequences could not be grouped because they encoded no protein and did not have 
characteristic terminal motifs. All identified transposons were combined into a common bean transposon library that includes 791 representative transposon sequences (Table 1 ) and is $2.12 \mathrm{Mb}$ in size.

\section{TRANSPOSON-RELATED EXPRESSED SEOUENCE TAGS (ESTs)}

A total of 148,267 ESTs from common bean were deposited in GenBank as of July 1, 2012. We hypothesized that some TEs may be transcriptionally active and that transposon-related ESTs may be detected. To test this, all ESTs were used to search against our common bean transposon database. A total of 5,328 ESTs were found to have significant sequence similarity to TEs $\left(E\right.$ value $\left.<10^{-5}\right)$. Excepting SINEs, each TE had EST hits though the numbers vary among the various TE superfamilies (Figure 1). We found 2,633 Ty-gypsy-related ESTs that account for $49.4 \%$ of the expressed TE sequences. A total of $546(10.2 \%)$ and $356(6.7 \%)$ ESTs were found to have significant similarity to Ty1-copia and unclassified LTRs, respectively. LINE elements are frequently transcribed and represent the most abundant TEs in mammals (Cordaux and Batzer, 2009). However, active LINEs are not common in plant genomes. It is interesting that we detected 893 (16.8\%) LINE-related ESTs which indicate that some LINEs are highly expressed in common bean. Furthermore, a total of 838 ESTs related to DNA transposons were found, including 322 Helitrons, 284 CACTAs, 156 MULEs, 72 hATs, and 4 PIF/Harbingers, which cumulatively represent $\sim 15.7 \%$ of the TE-related ESTs.

\section{STRUCTURAL ANALYSIS OF LTR RETROTRANSPOSONS}

Previous studies have shown that some plant LTR retrotransposons contain an extra ORF encoding envelope-like proteins, including two Ty1-copia elements, SIRE1 in soybean (Laten et al., 1998)

Table 1 | Summary of the common bean transposon database.

\begin{tabular}{cl}
\hline Transposon superfamily & Number of transposon in the database \\
\hline Class 1 & 285 \\
LTR retrotransposons & 176 \\
Ty1-copia & 73 \\
Ty3-gypsy & 80 \\
Unclassified & 23 \\
Non-LTR retrotransposon & 109 \\
LINE & 105 \\
SINE & 4 \\
Class 2 & 460 \\
CACTA & 348 \\
hAT & 23 \\
MULE & 45 \\
Helitron & 39 \\
PIF/Harbinger & 5 \\
Unclassified & 46 \\
Total & 791
\end{tabular}

and ToRTL1 in tomato (Tam et al., 2007), and three Ty3-gypsy elements, Cyclops2 in pea (Chavanne et al., 1998), Athila4 of Arabidopsis and Calypso of soybean (Wright and Voytas, 2002). To determine whether LTR retroelements of common bean carry an extra ORF coding envelope-like proteins, the internal regions of 65 Ty1-copia and 78 Ty3-gypsy families were annotated using FGE$\mathrm{NESH}^{2}$ and GENSCAN ${ }^{3}$. 96.9\% (63/65) of Tyl-copia and $80.8 \%$ (63/78) of Ty3-gypsy transposon families contain only retrotransposase encoding ORFs. Interestingly, two Ty1-copia families and 15 Ty-gypsy families were found to have an extra ORF located between the retrotransposase ORF and 3' LTR (Table 2; Figure 2). The extra ORFs do not share similarity with any retrotransposase sequences.

Two Ty1-copia families with the extra ORF, pvRetro3 and pvRetro4, shared less than 30\% similarity between their LTRs but their internal regions had $76 \%$ similarity over $5 \mathrm{~kb}$ indicating that these two elements likely diverged from a common ancestral LTR retroelement. To define the potential function of the extra ORFs, the 381-aa protein of pvRetro3 and 393-aa protein of pvRetro4 were used to search against GenBank and the Gypsy database ${ }^{4}$, however, no conserved domain was found for either of these two proteins. pvRetro3 and pvRetro4 were then used to perform BLASTN searches, the internal sequences from both elements share more than 65\% sequence similarity over $2 \mathrm{~kb}$ with that from MtRetro3 (AC144724:31185-43634) from Medicago truncatula; LjRetro3 (AP006091:15667-26573) in Lotus japonicas and Grm6 (gmp1-12a14-re-4, FJ198003) in soybean. It is interesting that MtRetro3 and LjRetro3 also contain two ORFs, the retrotransposase-encoded ORF (1597 and 1584-aa for MtRetro3 and LjRetro3, respectively) and an extra ORF (774 and 793-aa). However, Grm6 had only one ORF encoding a 1768aa retrotransposase. Notably, the 774-aa protein of MtRetro3 shows sequence similarity to the envelope-like proteins of SIRE1 $\left(E\right.$ value $\left.=6 \mathrm{e} \times 10^{-4}\right)$ and TorTL1 $\left(E\right.$ value $\left.=2 \mathrm{e} \times 10^{-10}\right)$. It also exhibits similarity to pvRetro3 $\left(1 \mathrm{e} \times 10^{-15}\right)$, pvRetro4 $\left(7 \mathrm{e} \times 10^{-16}\right)$, and LjRetro3 $\left(4 \mathrm{e} \times 10^{-16}\right)$. These results suggests that the pvRetro3 381-aa and pvRetro4 393-aa proteins were likely envelope-like proteins but have either diverged or been truncated.

We also found 15 Ty3-gypsy retrotransposon families that contained an extra ORF (ORF2) in addition to the ORF(s) that encode the retrotransposase (complete or partial; Figure 2; Table 2). Unlike the two Ty1-copia families and other retroelements with envelope-like proteins, the retrotransposase and the ORF2-encoded protein of all these 15 retroelements were in opposite transcriptional orientations. Furthermore, no detectable sequence similarity was found between described envelope proteins and the proteins encoded by the ORF2s. However, the ORF2-encoded proteins shared sequence similarity with each other suggesting a common origin. For instance, the protein of pval118d24-re-5 shows significant similarity to that of pvRetro13 $\left(1 \mathrm{e} \times 10^{-66}\right)$ and pvRetro124 $\left(2 \mathrm{e} \times 10^{-39}\right)$. In addition, some of the proteins contain a conserved domain, structural maintenance

\footnotetext{
${ }^{2}$ http://linuxl.softberry.com

${ }^{3}$ http://genes.mit.edu/GENSCAN.html

${ }^{4}$ http://gydb.org/index.php/Main_Page
} 


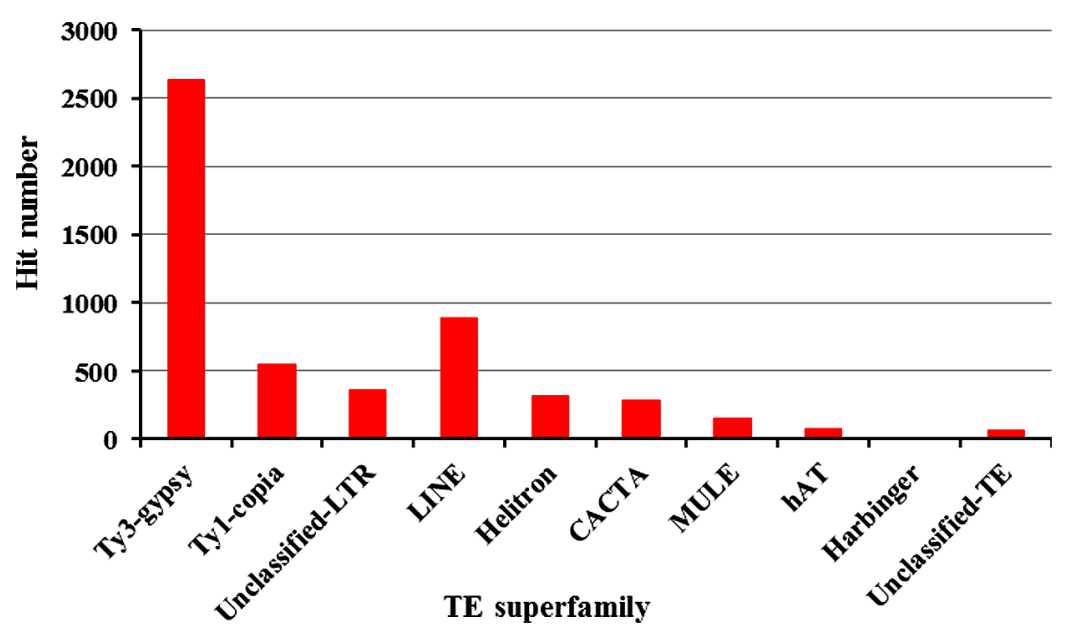

FIGURE 1 | Summary of common bean-derived ESTs that share sequence similarity with different transposons.

of chromosomes (SMC), that binds DNA and acts in organizing and segregating chromosomes for partitioning (Marchler-Bauer et al., 2011).

Open reading frame 2-encoded proteins were then used conduct TBLASTN searches to detect if homologous sequences were present in other genomes. Thirteen, 40, and 226 significant hits ( $E$ value $<1 \times 10^{-5}$ ) were found in G. max, M. truncatula and L. japonicas, respectively. All hits and their flanking sequences were manually inspected and a complete elements, gtd1-136a20-re-1
(FJ402912) from G. max and LjRetro26 (AP009775:48573-58015) from $L$. japonicas, were identified. No intact element was identified in M. truncatula suggesting complete element may have been degraded, missed by our investigation or was not captured in the sequence assembly. gtd1-136a20-re-1 and LjRetro26 have ORF2s encoding 868 and 461-aa protein, respectively. The two retrotransposons likely are autonomous elements as they encode $>1800$-aa retrotransposase containing all domains necessary for retrotransposition.

Table 2 | Common bean LTR retrotransposons with the envelope-like protein or an additional open reading frame (ORF2).

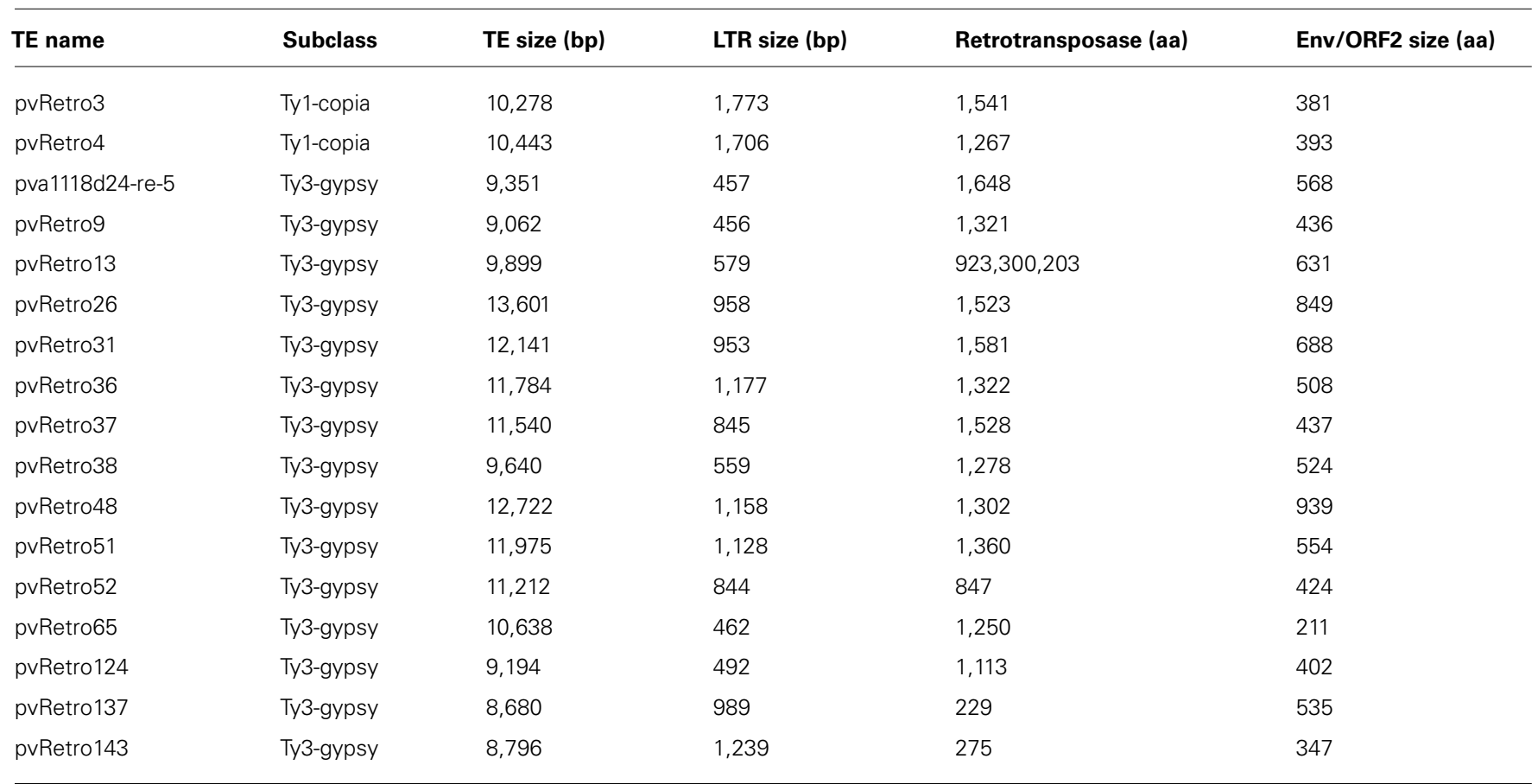

The proteins that are necessary for retrotransposition of pvRetro13 were encoded by three ORFs (Figure 2), but the internal sequence for the other 14 families contained only one ORF encoding the retrotransposase. 


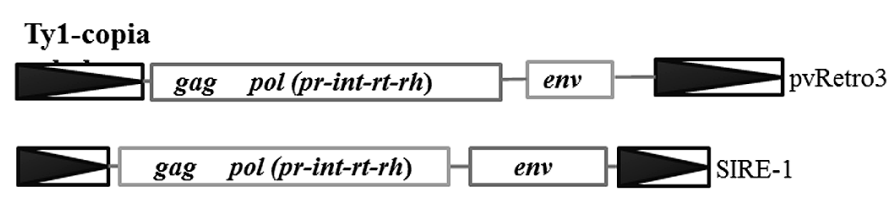

Ty3-gypsy
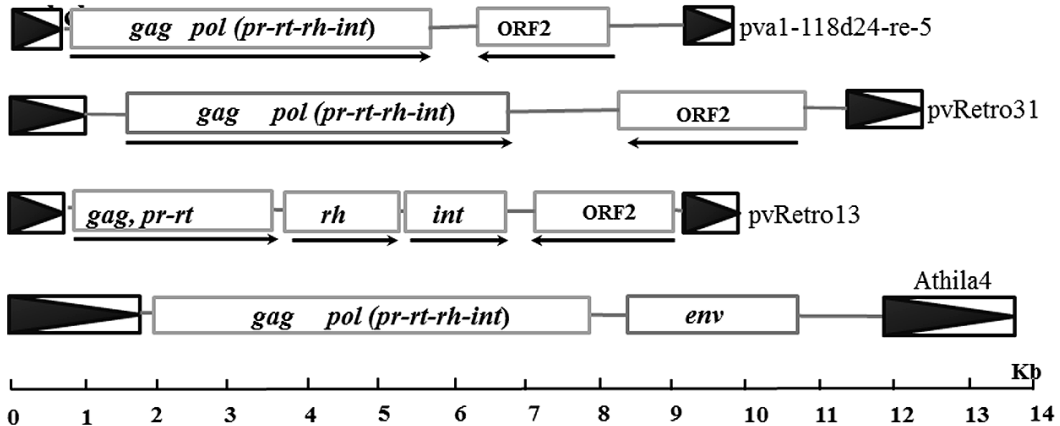

FIGURE 2 | Sequence structures of different LTR retrotransposons. Boxes with black triangles represent transposon LTRs. Rectangles indicated open reading frames (ORF) encoding retrotransposases or other proteins and arrows indicate the transcription orientations for retroelements with ORF2.

\section{PHYLOGENETIC ANALYSIS OF LTR RETROTRANSPOSONS}

To gain insight into evolutionary relationships between LTR retrotransposons from common bean and other plants, two phylogenetic trees were constructed based on conserved RT domains of LTR retrotransposons. Among 65 Ty1-copia families in common bean, 37 families carry nearly complete RT domains, whereas the other 28 families contain either short or no RT domains. The RT sequences of 56 Tyl-copia elements, including 37 families from common bean, 10 families from soybean, and 9 elements from other plants were used to build a phylogenetic tree. These retrotransposons were clustered into seven clades, clade I contains 19 elements whereas clade $\mathrm{V}$ only has pvRetro2 (Figure 3A). Two retroelements that encode envelope-like proteins, SIRE1 in soybean and TorTL1 in tomato, were grouped into clade II together with pvRetro3, pvRetro4, MtRetro3, and LjRetro3. Even though pvRetro3, pvRetro4, and SIRE1 fell into a same clade, pvRetro3, and pvRetro4 likely shared more recent ancestry with Grm6 from soybean than with SIRE1 as both pvRetro3 and pvRetro4 show higher sequence similarity with Grm6 than SIRE1 at the nucleotide level.

Another phylogenetic tree was made using RT domains of 48 Ty3-gypsy families including 30 common bean transposons and 5 soybean retroelements. The Ty3-gypsy retrotransposons were grouped into eight clades (Figure 3B). Clades IV and VI contain only one element, whereas, clade VIII contains 19 retroelements. It is worth to note that all 15 families containing ORF2 (Table 2) but pvRetro137 and pvRetro143 that do not encode RT domains were clustered into clade VIII. Three LTR retrotransposons encoding envelope-like proteins, Cyclops 2 in pea (Chavanne etal., 1998), Athila4 of Arabidopsis and Calypso in soybean (Wright and Voytas, 2002) fell into clade V. Interestingly, three elements, pvRetro11, pvRetro14, and pvRetro29 were classified together the centromeric retrotransposons, CRM in maize (Jin et al., 2004) and RIRE7 in rice (Kumekawa et al., 2001), and three elements, Gmr4, Gmr59, and Gmr215, which may be centromeric retroelements in soybean (Du et al., 2010a). pvRetro11, pvRetro14, and pvRetro29 were used to search against the soybean transposon database ${ }^{5}$ (Du et al., 2010b). The internal sequences of all three retroelements show $\sim 80 \%$ sequence similarity with Gmr4 over a 300-bp region but the LTRs from pvRetro11, pvRetro14 and pvRetro29 share no detectable similarity with Gmr4 or other any soybean transposon. To determine if pvRetro11, pvRetro14, and pvRetro29 are located in centromeric regions in common bean, the genomic distributions of three retrotransposons were investigated. Although each family varies in distribution, pvRetro11, pvRetro14, and pvRetro29 were dispersed across the common bean genome (Figure 4, chromosome 1 shown as illustrative of the other 10 chromosomes).

\section{DISCUSSION}

TEs are ubiquitous component in all eukaryotic genomes complicating assembly of whole genome shotgun sequencing projects and annotation of reference genomes. Although numerous computational tools have been developed that have accelerated the process of identifying transposons (Bergman and Quesneville, 2007), it still is a time-consuming and challenging task to annotate these abundant and often highly divergent elements. Using a single annotation approach, either homology- or structure-based, will render a biased transposon annotation. In this study, we annotated TEs in common bean by a combination of homology and structure approaches and developed a 2.12-Mb transposon database of common bean which includes 791 transposon reference sequences. Heretofore, only a few transposons of common bean had been described (Garber et al., 1999; Wawrzynski et al., 2008), thus the overwhelming majority of transposons in our database represent previously unrecognized TEs in common bean. We should note

${ }^{5}$ http://soybase.org/soytedb 


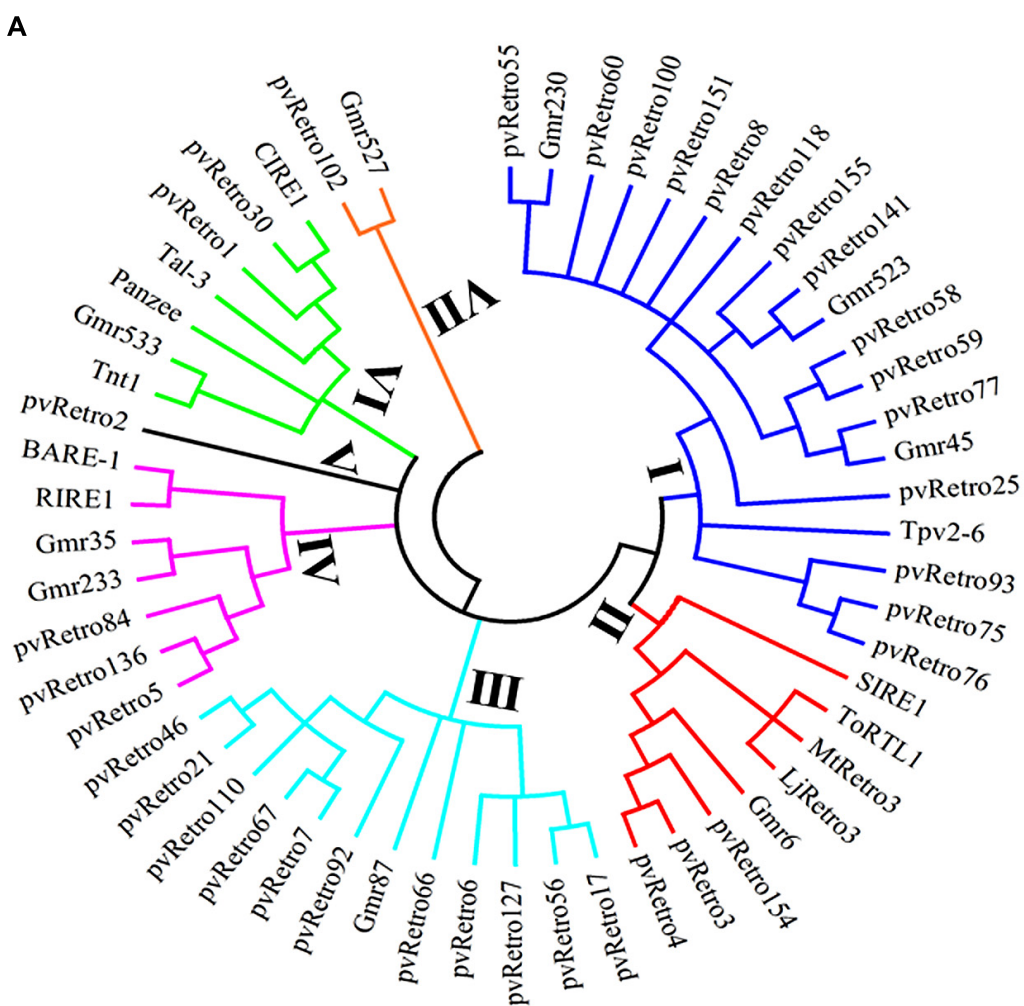

B

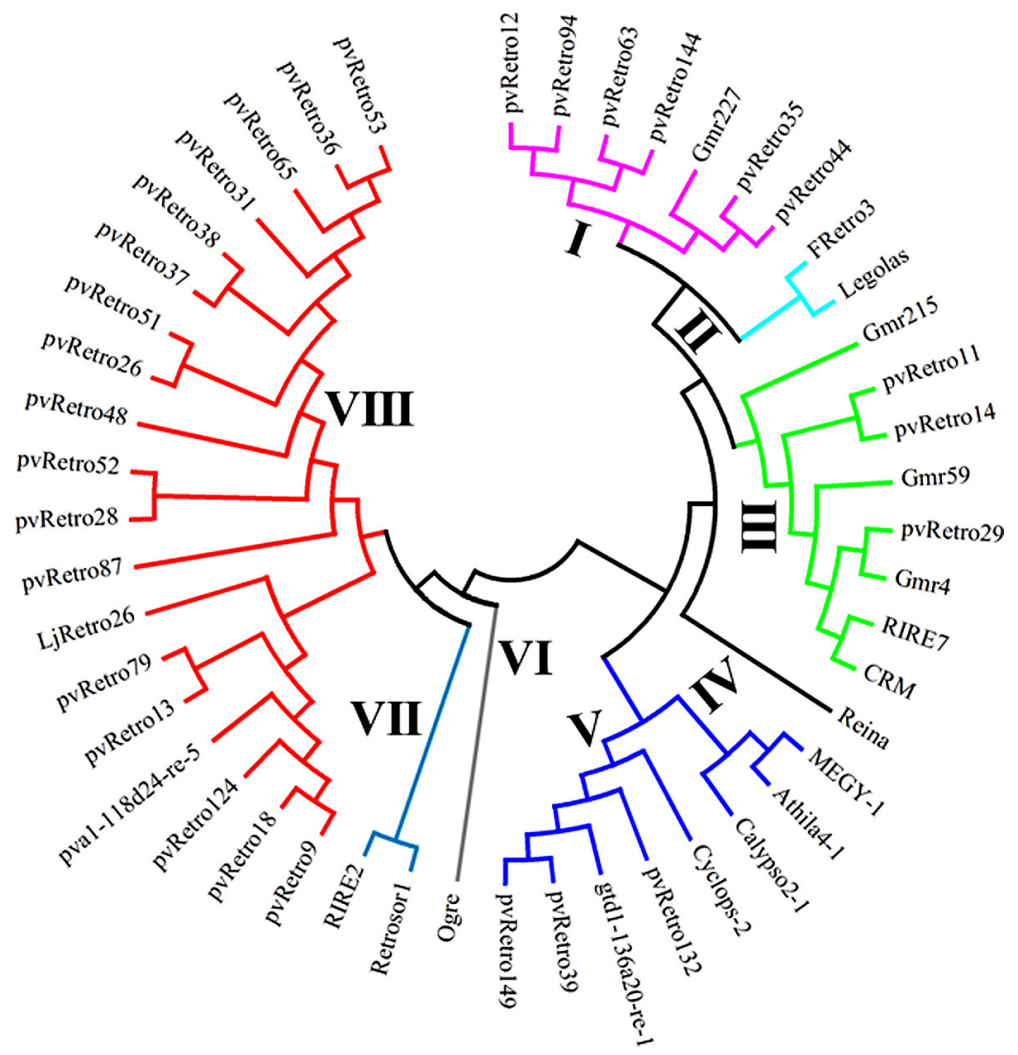

FIGURE 3 | Phylogenetic tree using the conserved RT domains of Ty1-copia (A) and Ty3-gypsy (B) LTR retrotransposons. 


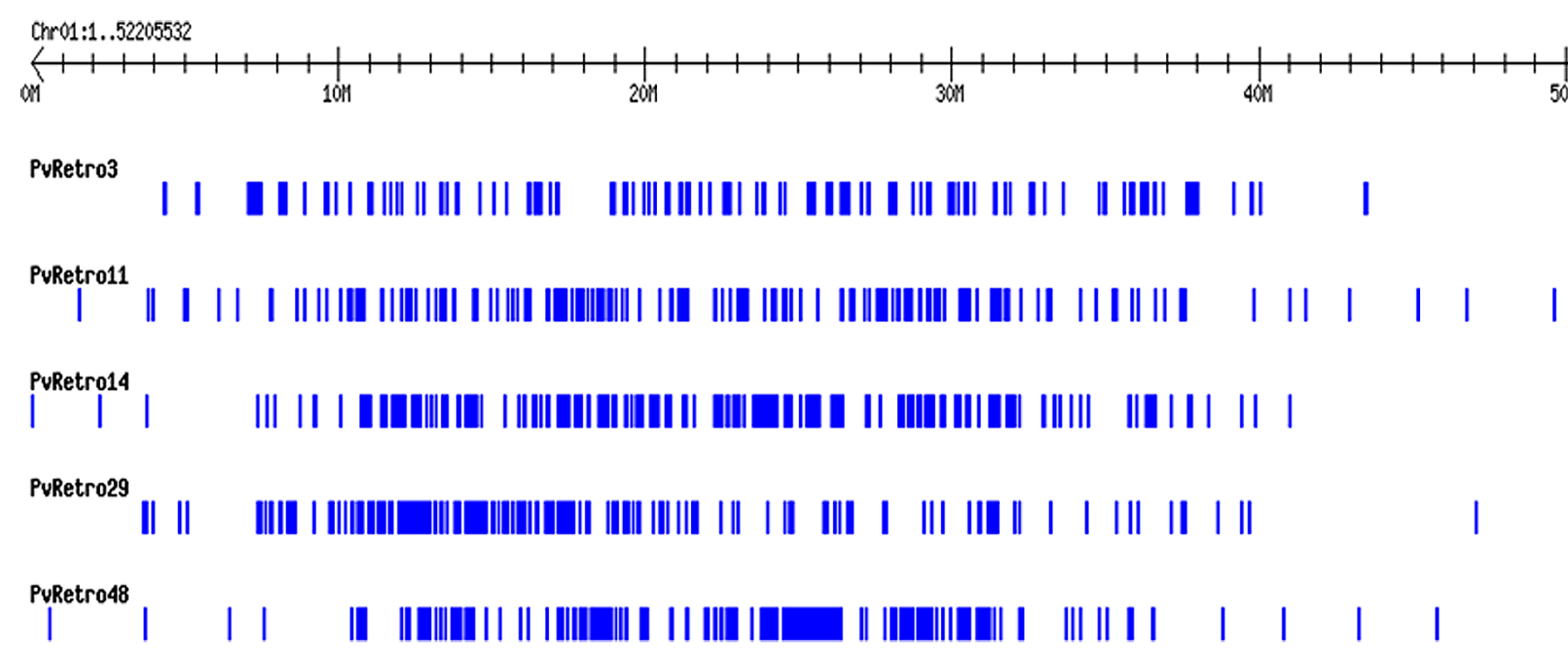

FIGURE 4 | Distribution of five LTR retroelement families on the chromosome $\mathbf{1}$ of common bean. The distribution patterns of the five transposons across other nine chromosomes are not shown as they are similar to chromosome 1.

that we do not include all members for each transposon family in the transposon database as it will enlarge the size of TE library and impede computational analyses.

Transposon databases are useful resources for both applied and basic research in plants (Ouyang and Buell, 2004; Du et al., 2010b). First, TEs are one of the most conspicuous genomic features in plants and have been shaping host genomes through insertions, deletions, and illegitimate recombination. TEs also provide raw material for the evolution of new genes and regulatory networks (Feschotte and Pritham, 2007; Cordaux and Batzer, 2009). Therefore, the transposon database is useful not only for genome annotation but also for the study of gene/genome evolution, domestication and population genetics in plants (Panaud, 2009). Second, a transposon library can aid in comparative analyses. Syntentic relationships between common bean and other legumes have been established (Lin et al., 2010; McClean et al., 2010; Bitocchi et al., 2012); however, it is still not understood what role structural rearrangements may have played in legume genome divergence and speciation. The availability of the genome sequences from soybean (Schmutz et al., 2010), pigeon pea (Varshney et al., 2011) and other legumes will allow us to compare the transposon organization and dynamics at genome level and to define the evolutionary events mediated by transposons. Third, the common bean transposons may be helpful for annotating other related genomes. Numerous transposons were identified in this study that are not restricted to the common bean as homologs can be found in soybean, M. truncatula, L. japonicas, and other genomes. Thus, the common bean transposon database offers a good resource to predict the transposons in closely related genomes. Finally, the transposon dataset may be used to identify active transposons in common bean. Active endogenous transposons can be used for insertional mutagenesis. For instance, the Ac/Ds elements and the Mutator transposons have been used for gene discovery in maize (Raizada et al., 2001; Vollbrecht et al., 2010). In this study, we identified 5,328 transposon-related ESTs. It may be that transposons have involved in gene construction and that these ESTs are genic fragments or there may be transcriptionally active transposons in common bean.

LTR retrotransposons containing envelope-like proteins are not common in plants. Legume genomes are an exception, however, as retroelements with envelope-like protein were identified in soybean (Laten et al., 1998; Wright and Voytas, 2002), pea (Chavanne et al., 1998) and L. japonicas (Holligan et al., 2006). In this study, we found two Ty1-copia families, pvRetro3 and pvRetro4, that contain an ORF encoding an envelope-like protein. We also identified retroelement MtRetro3 in M. truncatula and LjRetro3 in $L$. japonicas which both encode a protein showing sequence similarity to other envelope-like proteins. Phylogenetic analysis indicated that pvRetro3, pvRetro4, MtRetro3, and LjRetro3 were related to the SIRE1 element that encodes retroviral envelope-like protein in soybean (Laten et al., 1998). It is possible that the envelope-like proteins in common bean, and other legumes, may have a role in horizontal transfer of the LTR elements.

Of note were the 15 Ty3-gypsy families with an extra ORF (ORF2) encoding proteins in opposite transcriptional orientation to the retrotransposase (Table 2; Figure 2). In addition to retroelements with envelope-like proteins, the Retrosat2/FRetro3 family from rice also has an extra ORF, ORF0, located between $5^{\prime}$ LTR and retrotransposase that encodes the protein involved in metabolism (catabolic processes; Gao et al., 2009, 2012). The ORF2-encoded proteins do not share any sequence similarity with either envelope-like protein or the ORF0-protein of Retrosat 2 and phylogenetic analysis indicated that 15 retrofamilies with ORF2 were grouped into different clades from elements with the envelope-like protein (Figure 3B). These results indicate that the ORF2-encoded protein is likely not related to envelopelike proteins. The conserved SMC domain (Marchler-Bauer et al., 2011) was found in some ORF2-encoded proteins and may have a functional role in the biology of these transposons though 
that remains ambiguous. ORF2 proteins were also detected other legumes indicating an ancient origin before the divergence of the legumes.

\section{MATERIALS AND METHODS DATA SOURCES}

The common bean genome sequence, including chloroplast and mitochondrion, was generated by the common bean genome sequencing project (available at www.phytozome.org; Schmutz et al., 2014). Fifteen common bean BAC sequences, DQ205649 (Abdelnoor et al., 2006), DQ323045 (Kami et al., 2006), FJ817289FJ817291 (David et al., 2009), and GU215957-GU215966 (Lin et al., 2010), and EST sequences of common bean were downloaded from GenBank.

\section{SEQUENCE ANALYSIS}

We conducted transposon annotation using different approaches according to the sequence features of various transposon superfamilies. For annotating LTR retrotransposons, the genome sequence was screened with LTR_FINDER (Xu and Wang, 2007) using default settings except that we used a $50 \mathrm{bp}$ of minimum LTR length and $50 \mathrm{bp}$ of minimum distance between LTRs. LINEs were predicted by the retrotransposase and polyA motif. The SINEs were detected based on the polyA structure feature and combined with BLASTN searches. To annotate DNA transposons, the conserved domain of each superfamily (Wicker et al., 2007) was used to conduct TBLASTN against the genome sequence and hit sequences $\left(E\right.$-value $\left.<1 \times 10^{-20}\right)$ and flanking sequences $(10-\mathrm{kb}$ for each side) were extracted and used for BLASTN searches to define transposon boundaries. In addition, we used the MITEsHunter program (Han and Wessler, 2010) to identify small DNA transposons that do not encode proteins and may be missed by the transposase searches. All annotated transposons were conducted all against all BLAST searches to group them into different families by following previous publication (Wicker et al., 2007) or to remove the highly similar trtansposons. To simplify the transposon database, only the representative elements were included which are intact elements or longer transposon fragments and share more than $70 \%$ sequence similarity with other elements from same family or group.

\section{SEQUENCE STRUCTURE AND PHYLOGENETIC ANALYSIS OF LTR RETROTRANSPOSONS}

The sequence structures of LTR retrotransposons were predicted by the FGENESH ${ }^{6}$ and GENSCAN ${ }^{7}$ programs. The predicted proteins were used to search GenBank and the Gypsy database ${ }^{8}$ to identify functions and the RT conserved domains.

The phylogenetic trees were constructed based on the RT conserved domains of LTR retroelements from common bean, soybean, and other organisms. The alignments of multiple RT sequences were generated with CLUSTALW program ${ }^{9}$ with default options and the ambiguous regions were deleted. Phylogenetic

\footnotetext{
${ }^{6} \mathrm{http} / /$ linuxl.softberry.com

${ }^{7} \mathrm{http}: / /$ genes.mit.edu/GENSCAN.html

${ }^{8}$ http://gydb.org/index.php/Main_Page

${ }^{9}$ http://www.ebi.ac.uk/clustalw
}

trees were generated with MEGA $4{ }^{10}$ using the neighbor-joining method. A total of 22 retroelements from different legumes were use, including Cyclops-2 (AJ000640) and Ogre (AY299398) from pea (Pisum sativum L.); Panzee (AJ000893) from Pigeon pea (Cajanus cajan); MEGY-1 (AC146683) from M. truncatula and 15 elements from soybean. In addition to SIRE1-4 (AY205608) and Calypso2-1 (AF186183), 13 LTR retroelements in soybean, including nine Tyl-copia elements (Gmr6, Gmr35, Gmr45, Gmr87, Gmr230, Gmr233, Gmr523, Gmr527, and Gmr533) and four Ty3-gypsy elements (Gmr4, Gmr5, Gmr215, and Gmr227), were obtained from the soybean transposon database $^{11}$ (Du et al., 2010b). In addition, 14 retrotransposons from other plants also were selected which include Athila41(AC007209), Legolas (AC006570), and Ta1-3 (X13291) from Arabidopsis thaliana; RIRE2 (AB030283) and RIRE7 (AB033235) from rice (Oryza sativa); RIRE1 (D85597) from O. australiensis; FRetro3 (GU369679) from O. brachyantha; Reina (U69258), and CRM (AY129008) from maize (Zea mays); Retrosor1 (AF098806) from sorghum (Sorghum bicolor); BARE-1 (Z17327) from barley (Hordeum vulgare); Tnt1 (X13777) from tobacco (Nicotiana tabacum; CIRE1 (AM040263) from Citrus sinensis and ToRTL1 (U68072) from tomato (Lycopersicon esculentum).

\section{ACKNOWLEDGMENTS}

This research was funded by the National Science Foundation (DBI 0822258) and United States Department of Agriculture CSREES (2009-01860) to Scott A. Jackson. The work conducted by the U.S. Department of Energy Joint Genome Institute is supported by the Office of Science of the U.S. Department of Energy under Contract No. DE-AC02-05CH11231.

\section{REFERENCES}

Abdelnoor, R. V., Christensen, A. C., Mohammed, S., Munoz-Castillo, B., Moriyama, H., and Mackenzie, S. A. (2006) Mitochondrial genome dynamics in plants and animals: convergent gene fusions of a MutS homologue. J. Mol. Evol. 63, 165-173. doi: 10.1007/s00239-005-0226-9

Bao, Z., and Eddy, S. R. (2002). Automated de novo identification of repeat sequence families in sequenced genomes. Genome Res. 12, 1269-1276. doi: 10.1101/gr.88502

Bergman, C. M., and Quesneville, H. (2007). Discovering and detecting transposable elements in genome sequences. Brief. Bioinform. 8, 382-392. doi: 10.1093/bib/bbm048

Bitocchi, E., Nanni, L., Bellucci, E., Rossi, M., Giardini, A., Zeuli, P. S., et al. (2012). Mesoamerican origin of the common bean (Phaseolus vulgaris L.) is revealed by sequence data. Proc Natl Acad Sci U.S.A. 109, E788-E796. doi: 10.1073/pnas.1108973109

Blair, M. W., Hurtado, N., Chavarro, C. M., Muñoz-Torres, M. C., Giraldo, M. C., Pedraza, F., et al. (2011). Gene-based SSR markers for common bean (Phaseolus vulgaris L.) derived from root and leaf tissue ESTs: an integration of the BMc series. BMC Plant Biol. 11:50. doi: 10.1186/1471-2229-11-50

Brouha, B., Schustak, J., Badge, R. M., Lutz-Prigge, S., Farley, A. H., Moran, J. V., etal. (2003). Hot L1s account for the bulk of retrotransposition in the human population. Proc. Natl. Acad. Sci. U.S.A. 100, 5280-5285. doi: 10.1073/pnas.0831042100

Chavanne, F., Zhang, D. X., Liaud, M. F., and Cerff R. (1998). Structure and evolution of Cyclops: a novel giant retrotransposon of the Ty3/Gypsy family highly amplified in pea and other legume species. Plant Mol. Biol. 37, 363-375. doi: 10.1023/A:1005969626142

\footnotetext{
${ }^{10} \mathrm{http}: / /$ www.megasoftware.net

${ }^{11} \mathrm{http}: / /$ soybase.org/soytedb
} 
Cordaux, R., and Batzer, M. A. (2009). The impact of retrotransposons on human genome evolution. Nat. Rev. Genet. 10, 691-703. doi: 10.1038/nrg2640

David, P., Chen, N. W., Pedrosa-Harand, A., Thareau, V., Sevignac, M., Cannon, S. B., et al. (2009). A nomadic subtelomeric disease resistance gene cluster in common bean. Plant Physiol. 151, 1048-1065. doi: 10.1104/pp.109.142109

Du, J., Tian, Z., Hans, C. S., Laten, H. M., Cannon, S. B., Jackson, S. A., et al. (2010a). Evolutionary conservation, diversity and specificity of LTR-retrotransposons in flowering plants: insights from genome-wide analysis and multi-specific comparison. Plant J. 63, 584-598. doi: 10.1111/j.1365-313X.2010.04263.x

Du, J., Grant, D., Tian, Z., Nelson, R. T., Zhu, L., Shoemaker, R. C., et al. (2010b). SoyTEdb: a comprehensive database of transposable elements in the soybean genome. BMC Genomics 11:13. doi: 10.1186/1471-2164-11-113

Feschotte, C., Jiang, N., and Wessler, S. R. (2002). Plant transposable elements: where genetics meets genomics. Nat. Rev. Genet. 3,329-341. doi: 10.1038/nrg793

Feschotte, C., and Pritham, E. J. (2007). DNA transposons and the evolution of eukaryotic genomes. Annu. Rev. Genet. 41, 331-368. doi 10.1146/annurev.genet.40.110405.090448

Gao, D., Gill, N., Kim, H. R., Walling, J. G., Zhang, W., Fan, C., et al. (2009). A lineage-specific centromere retrotransposon in Oryza brachyantha. Plant J. 60 820-831. doi: 10.1111/j.1365-313X.2009.04005.x

Gao, D., Jimenez-Lopez, J. C., Iwata, A., Gill, N., and Jackson S. A. (2012) Functional and structural divergence of an unusual LTR retrotransposon family in plants. PLoS ONE 7:e48595. doi: 10.1371/journal.pone.0048595

Garber, K., Bilic, I., Pusch,O., Tohme, J., Bachmair, A., Schweizer, D., et al. (1999) The Tpv2 family of retrotransposons of Phaseolus vulgaris: structure, integration characteristics, and use for genotype classification. Plant Mol. Biol. 39, 797-807. doi: 10.1023/A:1006132532684

Grewal, S. I., and Jia, S. (2007). Heterochromatin revisited. Nat. Rev. Genet. 8, 35-46. doi: $10.1038 / \mathrm{nrg} 2008$

Han, Y., and Wessler, S. R. (2010). MITE-Hunter: a program for discovering miniature inverted-repeat transposable elements from genomic sequences. Nucleic Acids Res. 38:e199. doi: 10.1093/nar/gkq862

Holligan, D., Zhang, X., Jiang, N., Pritham, E. J., and Wessler, S. R. (2006). The transposable element landscape of the model legume Lotus japonicus. Genetics 174, 2215-2228. doi: 10.1534/genetics.106.062752

Jin, W., Melo, J. R., Nagaki, K., Talbert, P. B., Henikoff, S., Dawe, R. K., et al. (2004). Maize centromeres: organization and functional adaptation in the genetic background of oat. Plant Cell 16, 571-581. doi: 10.1105/tpc.018937

Juretic, N., Bureau, T. E., and Bruskiewich, R. M. (2004). Transposable element annotation of the rice genome. Bioinformatics 20, 155-160. doi: 10.1093/bioinformatics/bth019

Kami, J., Poncet, V., Geffroy, V., and Gepts, P. (2006). Development of four phylogenetically-arrayed BAC libraries and sequence of the APA locus in Phaseolus vulgaris. Theor. Appl. Genet. 112, 987-998. doi: 10.1007/s00122-005-0201-2

Kojima, K. K. (2010). Different integration site structures between L1 proteinmediated retrotransposition in cis and retrotransposition in trans. Mob. DNA 1:17. doi: $10.1186 / 1759-8753-1-17$

Kumekawa, N., Ohmido, N., Fukui, K., Ohtsubo, E., and Ohtsubo H. (2001). A new gypsy-type retrotransposon, RIRE7: preferential insertion into the tandem repeat sequence $\mathrm{Trs} \mathrm{D}$ in pericentromeric heterochromatin regions of rice chromosomes. Mol. Genet. Genomics 265, 480-488. doi: 10.1007/s004380000436

Laten, H. M., Majumdar, A., and Gaucher, E. A. (1998). SIRE-1, a copia/Ty1-like retroelement from soybean, encodes a retroviral envelope-like protein. Proc. Natl. Acad. Sci. U.S.A. 95, 6897-6902. doi: 10.1073/pnas.95.12.6897

Lin, J. Y., Stupar, R. M., Hans, C., Hyten, D. L., and Jackson, S. A. (2010) Structural and functional divergence of a $1-\mathrm{Mb}$ duplicated region in the soybean (Glycine max) genome and comparison to an orthologous region from Phaseolus vulgaris. Plant Cell 22, 2545-2561. doi: 10.1105/tpc.110. 074229

Marchler-Bauer, A., Lu, S., Anderson, J. B., Chitsaz, F., Derbyshire, M. K., DeWeese-Scott, C., et al. (2011). CDD: a Conserved Domain Database for the functional annotation of proteins. Nucleic Acids Res. 39, D225-D229. doi: $10.1093 /$ nar/gkq1189
McCarthy, E. M., and McDonald, J. F. (2003). LTR_STRUC: a novel search and identification program for LTR retrotransposons. Bioinformatics 19, 362-367. doi: 10.1093/bioinformatics/btf878

McClean, P. E., Mamidi, S., McConnell, M., Chikara, S., and Lee, R. (2010). Synteny mapping between common bean and soybean reveals extensive blocks of shared loci. BMC Genomics 11:184. doi: 10.1186/1471-2164-11-184

Ouyang, S., and Buell C. R. (2004). The TIGR Plant Repeat Databases: a collective resource for the identification of repetitive sequences in plants. Nucleic Acids Res. 32, D360-D363. doi: 10.1093/nar/gkh099

Panaud, O. (2009). The molecular bases of cereal domestication and the history of rice. C. R. Biol. 332, 267-272. doi: 10.1016/j.crvi.2008.09.003

Raizada, M. N., Nan, G. L., and Walbot, V. (2001). Somatic and germinal mobility of the RescueMu transposon in transgenic maize. Plant Cell 13, 1587-1608. doi: 10.1105/tpc.13.7.1587

Roulin, A., Piegu, B., Wing, R. A., and Panaud, O. (2008). Evidence of multiple horizontal transfers of the long terminal repeat retrotransposon RIRE1 within the genus Oryza. Plant J. 53, 950-959. doi: 10.1111/j.1365-313X.2007.03388.x

Schmutz, J., Cannon, S. B., Schlueter, J., Ma, J., Mitros, T., Nelson, W., et al. (2010). Genome sequence of the palaeopoly- ploid soybean. Nature 463, 178-183. doi: 10.1038 /nature 08670

Schmutz, J., McClean, P. E., Mamidi, S., Wu, G. A., Cannon, S. B., Grimwood, J., et al. (2014). A reference genome for common bean and genome-wide analysis of dual domestications. Nat. Genet. 46, 707-713. doi: 10.1038/ng.3008

Tam, S. M., Causse, M., Garchery, C., Burck, H., Mhiri, C., and Grandbastien, M. A. (2007). The distribution of copia-type retrotransposons and the evolutionary history of tomato and related wild species. J. Evol. Biol. 20, 1056-1072. doi: 10.1111/j.1420-9101.2007.01293.x

Varshney, R. K., Chen, W., Li, Y., Bharti, A. K., Saxena, R. K., Schlueter, J. A., et al. (2011). Draft genome sequence of pigeonpea (Cajanus cajan), an orphan legume crop of resource-poor farmers. Nat. Biotechnol. 30, 83-89. doi: 10.1038/nbt.2022

Vollbrecht, E., Duvick, J., Schares, J. P., Ahern, K. R., Deewatthanawong, P., Xu, L., et al. (2010). Genome-wide distribution of transposed dissociation elements in maize. Plant Cell 22, 1667-1685. doi: 10.1105/tpc.109.073452

Wawrzynski, A., Ashfield, T., Chen, N. W., Mammadov, J., Nguyen, A., Podicheti, R., etal. (2008). Replication of nonautonomous retroelements in soybean appears to be both recent and common. Plant Physiol. 148, 1760-1771. doi: 10.1104/pp.108.127910

Wicker, T., Sabot, F., Hua-Van, A., Bennetzen, J. L., Capy, P., Chalhoub, B., et al. (2007). A unified classification system for eukaryotic transposable elements. Nat. Rev. Genet. 8, 973-982. doi: 10.1038/nrg2165

Wright, D. A., and Voytas, D. F. (2002). Athila4 of Arabidopsis and Calypso of soybean define a lineage of endogenous plant retroviruses. Genome Res. 12, 122-131. doi: 10.1101/gr.196001

Xu, Z., and Wang, H. (2007). LTR_FINDER: an efficient tool for the prediction of full-length LTR retrotransposons. Nucleic Acids Res. 35, W265-W268. doi: $10.1093 / \mathrm{nar} / \mathrm{gkm} 286$

Conflict of Interest Statement: The authors declare that the research was conducted in the absence of any commercial or financial relationships that could be construed as a potential conflict of interest.

Received: 03 June 2014; accepted: 25 June 2014; published online: 11 July 2014. Citation: Gao D, Abernathy B, Rohksar D, Schmutz J and Jackson SA (2014) Annotation and sequence diversity of transposable elements in common bean (Phaseolus vulgaris). Front. Plant Sci. 5:339. doi: 10.3389/fpls.2014.00339

This article was submitted to Plant Genetics and Genomics, a section of the journal Frontiers in Plant Science.

Copyright (C) 2014 Gao, Abernathy, Rohksar, Schmutz and Jackson. This is an openaccess article distributed under the terms of the Creative Commons Attribution License (CC BY). The use, distribution or reproduction in other forums is permitted, provided the original author $(s)$ or licensor are credited and that the original publication in this journal is cited, in accordance with accepted academic practice. No use, distribution or reproduction is permitted which does not comply with these terms. 